\title{
Homozygous Familial Hypercholesterolaemia with Valvular Aortic Stenosis and Significant Coronary Artery Disease: A Case Report
}

\author{
B Dutta, AKMM Islam, M Ullah, A Zaman, KK Karmakar, MZ Rahman, SK Kundu \\ Department of Cardiology, NICVD, Dhaka
}

\begin{abstract}
:
Key Words:

Homozygous Familial Hypercholesterolaemia is a genetic disorder which usually presents with early

Homozygous

Familial cardiovascular disease ranging from premature ischaemic disease, including myocardial infarction to

Hypercholesterolaemia,

Genetic disorder,

Myocardial

infarction,

aortic root stenosis. A 21 year old Bangladeshi male presented with exertional chest pain and breathlessness.

He was diagnosed as a case of Homozygous Familial Hypercholesterolaemia. His angina symptoms were due to underlying valvular aortic stenosis which is a rare presentation of Homozygous Familial Hypercholesterolaemia.
\end{abstract}

Valvular aortic

(Cardiovasc. j. 2014; 6(2): 180-183)

stenosis.

\section{Introduction:}

Familial Hypercholesterolaemia is a genetic disorder which presents with elevated levels of low density lipoproteins (LDL-C) and total serum cholesterol. The disease occurs due to an underlying genetic defect involving the short arm of chromosome 19. Mutations may result either in complete absence of the LDL receptor, defective binding of LDL to the receptor or defective internalization and transport of the LDL. There is a gene dose effect; homozygous individuals with two mutated LDL receptor alleles are more frequently and severely affected than heterozygote individuals who have a single mutant allele. ${ }^{1}$

The prevalence of heterozygous form is 1 in 500, with a higher ratio in certain populations. The homozygous form occurs in 1 in million. ${ }^{2}$ Patient with homozygous condition usually presents at an earlier age with signs and symptoms of ischaemic heart disease and peripheral vascular disease. ${ }^{3}$ Homozygotes with Familial Hypercholesterolaemia have a higher risk of aortic stenosis due to atherosclerotic involvement of the aortic root or the supravalvular region; but the incidence is relatively lower in heterozygotes. ${ }^{4}$

Premature atherosclerosis in Familial Hypercholesterolaemia may affect the aortic root, but involvement of the aortic valve is a peculiar feature. ${ }^{5}$ Premature aortic calcifications may also be present in adults presenting with Familial Hypercholestrolaemia. Individuals having valvular aortic stenosis may present with chest pain, syncopal attacks, dizziness or clinical features of heart failure.

\section{Case Presentation}

A 21-year-old male presented in the OutPatientDepartment with complaints of Exertional chest pain for 2 years and shortness of breath for 6 months. The pain was severe in intensity, radiated to the jaw and was relieved by rest. He also mentioned for eruptive skin lesion for last 7 years. There was no history of Rheumatic fever. There was no history of cough, palpitation, orthopnoea, syncope, sore throat or arthralgias. He did not smoke and denied any illicit drug use. He has one brother. His parents are alive and all his family members are healthy. No member of his maternal or paternal family had this sort of illness.

On physical examination, his pulse rate was $88 /$ minute, slow-rising, low-volume, regular in rhythm. His blood pressure was $100 / 70 \mathrm{~mm}$ of Hg. There was no pallor, jaundice, cyanosis, clubbing and JVP was not raised. On examination of his hand, xanthomatous lesions present over both eyelids, back of neck, back of elbows, knuckles, interdigital webs on both hands and had been there since the patient was 14 years of age (Figure-1\& 2).

Address of Correspondence : Dr. Bijoy Dutta, Department of Cardiology, NICVD, Dhaka, Bangladesh. email: bijoy_k51@yahoo.com. 


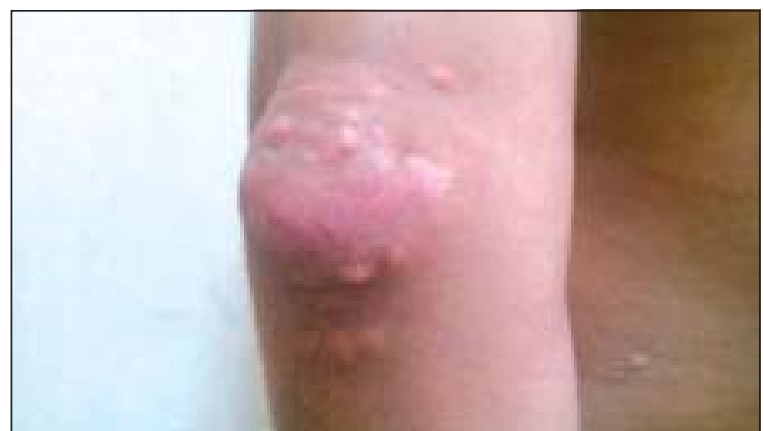

Fig.-1: Xanthomatous lesions back of elbows.

On palpation of precordium apex was located in left $5^{\text {th }}$ ICS just medial to midclavicular line and heaving in character. Systolic thrill present over Aortic area.On auscultation, first heart sound were normal, aortic component of second heart sound was soft. There was an ejection systolic murmur of grade 4 in aortic area which radiates to neck, best heard in patient sitting position leaning forward with breath hold after expiration. Other systemic examination revealed no abnormality. Peripheral pulses were palpable.

His Fasting Lipid Profile showed serum cholesterol level $588 \mathrm{mg} / \mathrm{dl}(150 \mathrm{mg} / \mathrm{dl}$ to $170 \mathrm{mg} /$ dl), LDL level - $424 \mathrm{mg} / \mathrm{dl}(<130 \mathrm{mg} / \mathrm{dl})$, triglycerides $178 \mathrm{mg} / \mathrm{dl}(50 \mathrm{mg} / \mathrm{dl}$ to $150 \mathrm{mg} / \mathrm{dl})$ and HDL level $27 \mathrm{mg} / \mathrm{dl}$ (40-60 mg/dl). EKG showed left ventricular hypertrophy with strain pattern. The patient was admitted and scheduled for cardiac imaging studies. Echocardiography showed thickened and calcified aortic valve leaflets with severe aortic stenosis at valvular level (peak gradient of $100 \mathrm{mmHg}$ ). Moderate concentric left ventricular hypertrophy was also seen with good biventricular systolic function. CAG revealed $70 \%$ stenosis in mid LAD.

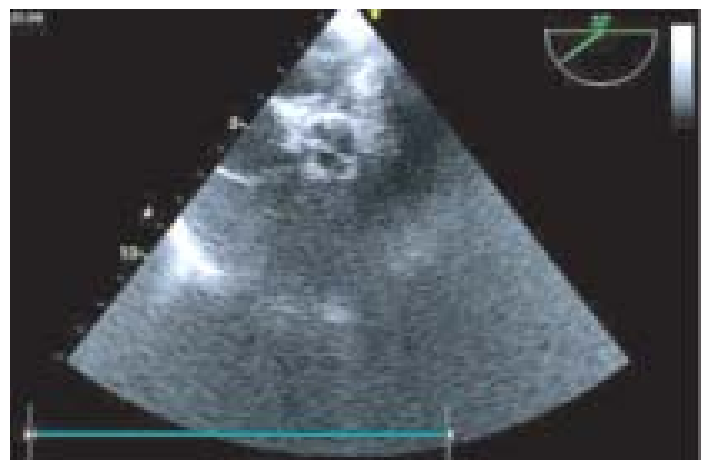

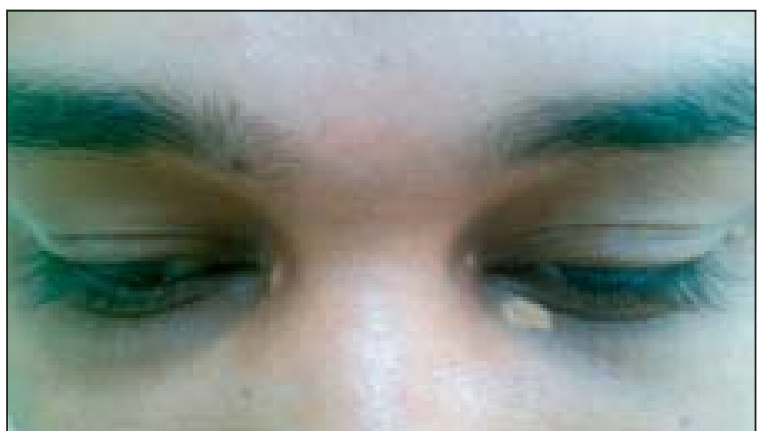

Fig.-2: Xanthomatous lesions present over both eyelids.

The patient's family was also screened by physical examination and a fasting lipid profile. Both his parents had elevated serum cholesterol levels and serum LDL levels with slightly elevated triglycerides and normal HDL levels. Thus on the basis of history, physical examination showing xanthomata, findings of Echocardiogram and results of lipid profile of the patient and his family, he was diagnosed as a case of homozygous Familial Hypercholestremia (type II hyperlipoproteinemia) with valvular aortic stenosis with significant CAD. Secondary causes such as nephrotic syndrome and hypothyroidism were excluded.

The patient was advised strict diet control (as per ATP III guideline). Treatment with atorvastatin was started with $40 \mathrm{mg} /$ day. Baseline liver function tests and creatinine kinase were done before initiation of therapy with statins. Metoprolol and aspirin were prescribed for CAD. The patient is currently awaiting aortic valve replacement with $\mathrm{CABG}$ surgery to correct his valvular aortic stenosis and significant CAD.

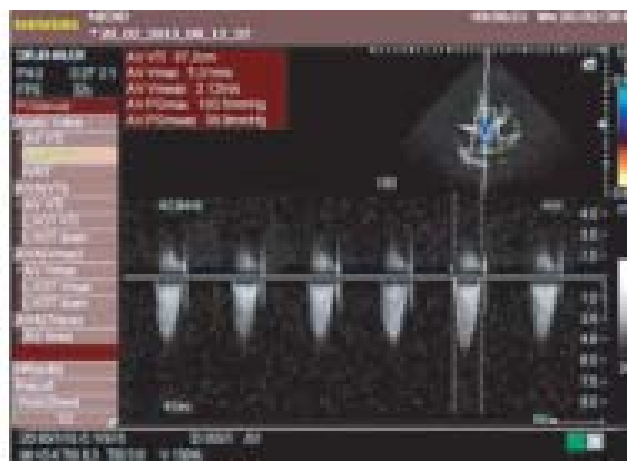

Fig.-3: Transthoracic and Transesophageal echocardiography showing severe Aortic stenosis. 
The patient is followed up regularly in the outpatient department and lipid profile is repeated every 3 months. Since his initial visit, his total serum cholesterol has decreased by $32 \%$ and his LDL level has decreased by $20 \%$. Ezetemibe $10 \mathrm{mg} /$ day was added. The goal is to bring down the cholesterol level to less than 200 $\mathrm{mg} / \mathrm{dl}$ and LDL level to at least below $130 \mathrm{mg} / \mathrm{dl}$ which are near optimal values as recommended by ATP III guidelines. This would lead to an improvement in the cholesterol to HDL ratio which is the most important predictor of atherosclerosis. The patient is monitored for any adverse effects of the drug therapy and liver function tests are scheduled at regular intervals.

\section{Discussion:}

Familial Hypercholesterolaemia is a genetic disease with hereditary transmission, but it presents with a wide range of phenotypic expressions. The variability is the result of various types of underlying mutation in the LDL receptor gene all of which result in Familial Hypercholesterolaemia. Risk factors such as age, gender, smoking and hypertension also contribute to the clinical signs and symptoms seen in the patient at the time of the onset of the disease. ${ }^{6}$

The typical manifestation seen in homozygous Familial Hypercholestrolaemia patients are coronary ostial stenosis and aortic root stenosis which occur due to cholesterol deposition in the aortic root at a young age. ${ }^{7}$ Contrary to homozygous Familial Hypercholesterolaemia, in the heterozygous strain the usual age of presentation is highly variable and depends on the molecular defect in the LDL receptor gene. Patients with coexisting cardiac disease risk factors may present at an earlier age with symptoms of cardiovascular disease. Most common cardiovascular presentation in homozygous Familial Hypercholestraemia is premature ischaemic heart disease, but relatively few cases present with earlier anginal symptoms due to atherosclerotic involvement of aortic root or supravalvular aortic stenosis. ${ }^{8}$

There should be a high index of clinical suspicion in considering valvular heart disease when chest pain is reported with clinical and laboratory features suggesting homozygous Familial Hypercholestrolaemia in young adults. An earlier intervention of valvular aortic stenosis along with lipid-lowering therapy decreases morbidity and mortality. ${ }^{9}$ Guyton JR et al reported in their study that a combination treatment with statin, ezetimibe and extendedrelease niacin may prove to be more beneficial than any of these drugs used alone. ${ }^{10}$ Statin therapy also has the added advantage of improving endothelial dysfunction in Familial Hypercholesterolaemia patients.

Invasive procedures such as lipid apheresis and liver transplant may be a last resort in patients who have no functional LDL receptors and are not responding to pharmacological therapy. Both procedures are expensive and have limited availability. Patients presenting with cardiac symptoms may need CABG or surgery involving the aortic root. ${ }^{11}$

\section{Conclusion:}

Valvular aortic stenosis should be considered a possible diagnosis in young adults presenting with angina symptoms with clinical and laboratory features suggestive of homozygous Familial Hypercholestrolaemia. Early diagnosis and intervention may lead to decrease in morbidity and mortality.

\section{References:}

1. Rader DJ, Cohen J, Hobbs HH. Monogenic hypercholesterolemia: new insights in pathogenesis and treatment. J Clin Invest 2003; 111: 1795-1803.

2. Goldberg AC, Hopkins PN, Toth PP, et al. Familial hypercholesterolemia: screening, diagnosis and management of pediatric and adult patients: clinical guidance from the National Lipid Association Expert Panel on Familial Hypercholesterolemia. Journal of Clinical Lipidology 2011; 5 (3 Suppl): S1-8.

3. Alrasadi K, Alwaili K, Awan Z, Valenti D, Couture P, Genest J. Aortic calcifications in familial hypercholesterolemia: potential role of the low density lipoprotein receptor gene. Am Heart J 2009; 157: 170-176.

4. Rallidis L, Naoumova RP, Thompson GR, Nihoyannopoulos P. Extent and severity of atherosclerotic involvement of the aortic valve and root in familial hypercholesterolaemia. Heart 1998; 80: 583-590. 
5. Kawaguchi A, Miyatake K, Yutani C, et al. Characteristic cardiovascular manifestation in homozygous and heterozygous familial hypercholesterolemia. Am Heart $J$ 1999; 137: 410-418.

6. Hill J, Hayden M, Frohlich J, Pritchard P. Genetic and environmental factors affecting the incidence of coronary artery disease in heterozygous familial hypercholesterolemia. Arterioscler Thromb Vasc Biol 1991; 11: 290-297.

7. Haitas B, Baker SG, Meyer TE, Joffe BI,Seftel HC. Natural history and cardiac manifestations of homozygous familial hypercholesterolaemia. QJM 1990; 76: 731-740.
8. Arora G, Fraser CD, Kearney DL, Vincent JA. Severe supravalvar aortic stenosis in familial homozygous hypercholesterolemia. Pediatr Cardiol 2006; 27: 282-285.

9. McKenney JM. Pharmacologic options for aggressive lowdensity lipoprotein cholesterol lowering: benefits versus risks. Am J Cardiol 2005; 96: 60E-66E.

10. Guyton JR, Brown BG, Fazio S, Polis A, Tomassini JE, Tershakovec AM. Lipid-Altering Efficacy and Safety of Ezetimibe/Simvastatin Coadministered With ExtendedRelease Niacin in Patients With Type IIa or Type IIb Hyperlipidemia. J Am Coll Cardiol 2008; 51: 1564-1572.

11. Qaiser S, Sheikh S, Malik MS. Homozygous Familial Hypercholestrolaemia presents with supravalvular aortic stenosis, a case report. J Pak Med Asso 2012;62: 612-614. 